\title{
Enhancing the Accuracy of Multimodal Biometric Systems
}

\author{
Meena Tiwari ${ }^{a}$, Dr. G. Santhi ${ }^{b}$, Dr. Ashish Mishra ${ }^{c}$ \\ ${ }^{a}$ Research Scholar, SRK, University, Bhopal (M.P.) India \\ ${ }^{\mathrm{b}}$ Professor SRK University Bhopal (M.P.) India \\ 'Professor Gyan Ganga Institute of Technology and Science Jabalpur (M.P.) ) India \\ aphmeenatiwari@gmail.com, bshan_v2006@yahoo.com, ${ }^{\mathrm{c}}$ ashish.mish2009@gmail.com
}

Article History: Received: 10 November 2020; Revised 12 January 2021 Accepted: 27 January 2021; Published online: 5 April 2021

\begin{abstract}
Biometric acknowledgment frameworks have progressed altogether in the most recent decade and their utilization in explicit applications will increment sooner rather than later. The capacity to direct important correlations and evaluations will be urgent to fruitful organization and expanding biometric selection. Indeed, even the best methodology and unimodal biometric frameworks couldn't completely address the issue of exactness and execution as far as their bogus acknowledge rate (FAR) and bogus oddball rate (FRR). In spite of the fact that multimodal biometric frameworks had the option to moderate a portion of the restrictions experienced in unimodal biometric frameworks, like non-all inclusiveness, uniqueness, nonadequacy, loud sensor information, parody assaults, and execution, the issue of low exactness actually continues. In this paper, we survey research papers zeroed in on the precision improvement in data combination of face and finger impression biometric acknowledgment frameworks, decide the primary highlights of the chose techniques, and afterward call attention to their benefits and inadequacies. We propose a novel methodology in relieving the issue of exactness and execution of data combination of multimodal biometric frameworks. This methodology utilizes multilayer perceptron neural organizations in preparing and testing of the organization while additionally proposing the utilization of the most well-known utilized unique mark in biometric field.
\end{abstract}

Keywords: C.I, multilayer perceptron, NN, fingerprint, Gabor filters, feature extraction, Fuzzy C-means.

\section{Introduction}

Biometrics is the robotized acknowledgment of people dependent on their physiological and social qualities [4] In the new years, biometric acknowledgment has been viewed as the most exact and dependable methods for confirmation contrasted with the customary methods of verification [2]. Biometric acknowledgment frameworks will in general tackle a portion of the issues experienced by customary validation strategies, for instance, the utilization of secret phrase and token. Because of the quick development of systems administration, correspondence, and versatility in the present electronically wired and unwired society which has prompted increment in crime percentage, the utilization of biometric acknowledgment frameworks has contributed significantly in tackling a portion of the issues experienced in regions of security, protection, unwavering quality, and so on.

With the increment in the reception of biometric acknowledgment frameworks, the requirement for precise and exceptionally performing biometric acknowledgment frameworks is required [17]. Because of the way that each biometric methodology has its limits and no biometric framework is awesome, this has raised an assortment of issues experienced in biometric frameworks by numerous scientists including:

(1) Uniqueness: Each person must have his/her own unique feature or characteristic different from another person;

(2) Universality: Every individual must possess this characteristic;

(3) Permanence: This basically implies that the one of a kind highlights found in the individual ought to be invariant after some time and highlights extricated ought to accordingly be steady and not be alterable over the long run, even the maturing of the individual ought not influence the element separated or vector;

(4) Performance: The presentation of biometric frameworks is given by the exactness of the frameworks. Bogus dismissal rate (FRR) and bogus acknowledgment rate (FAR) are two generally utilized standard measurements of the exactness of biometric frameworks [5]. There should be low bogus dismissal rate (FRR) and low bogus acknowledgment rate (FAR) 
(5) Protection from Circumvention: In request to give added security, a framework should be more enthusiastically to bypass than existing character the executives frameworks;

(6) Collectability: An individual's interesting actual attributes should be gathered in a sensibly simple design for brisk distinguishing proof Acceptability: The catch interaction gives a helpful estimation easily and is viewed as inconspicuous for the information subjects.

In order to overcome some of these problems mentioned above and to greatly increase the level of accuracy and performance, multimodal biometric systems are used [1].

\section{Fingerprint Recognition System.}

Special imprint affirmation is conceivably the most prominent biometrics. Finger impression affirmation is the effortlessness of acquirement, set up use and affirmation when stood out from other biometrics, and the path that there are different wellsprings of this biometric on each individual. Minutia control insinuates toward the successful component close to the end motivation behind the edge part. The best way to deal with balance fingerprints is with break down all image information on the fingerprints. Survey all image information requires a great deal of data, and this is inappropriate to making a promoted structure. Real showcased structures don't store the finger impression itself, in any case characteristics of the fingerprints, and codes related to the circumstance of these motivations behind properties. While simply ascribes are taken care of, they can't be reestablished as finger impression picture and consequently can't be used as assertion in legal workplaces. Novel imprint organizing is the cycle used to check whether two game plans of finger impression come from a comparable finger [12]. One finger impression is taken care of in the informational index and other is a laborer's current exceptional imprint.

\section{Fingerprint Recognition Steps}

Figure 1 shows fingerprint matching steps and figure 2 shows the fingerprint authentication steps.

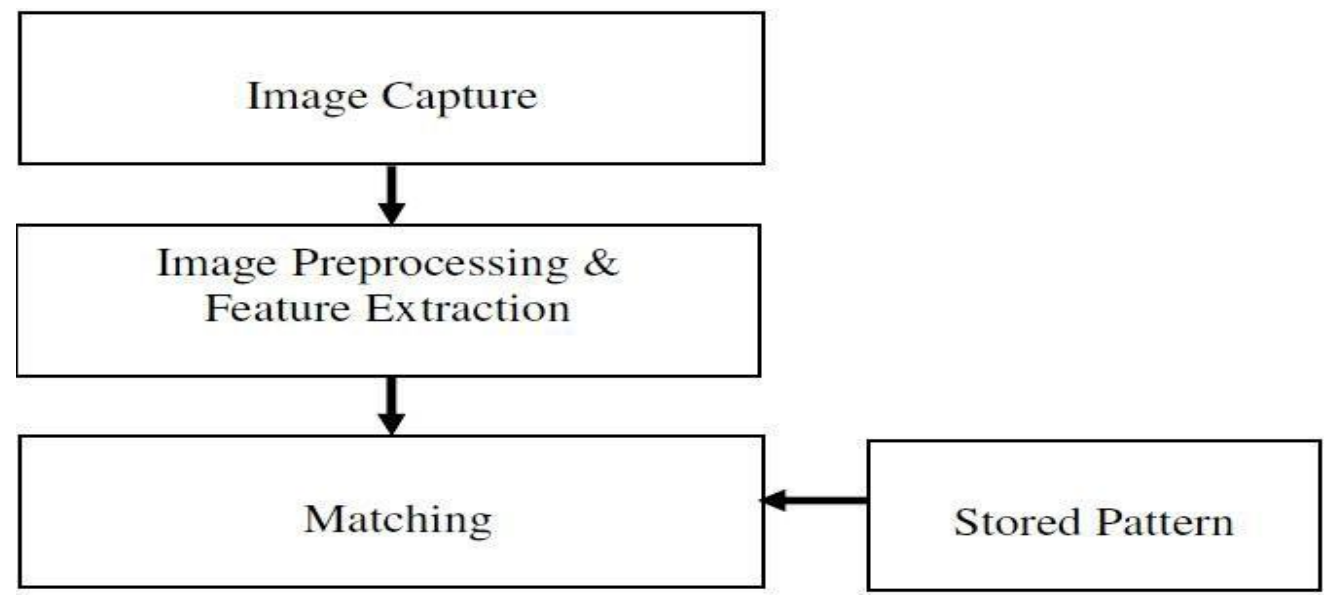

Figure 1: Fingerprint matching steps.

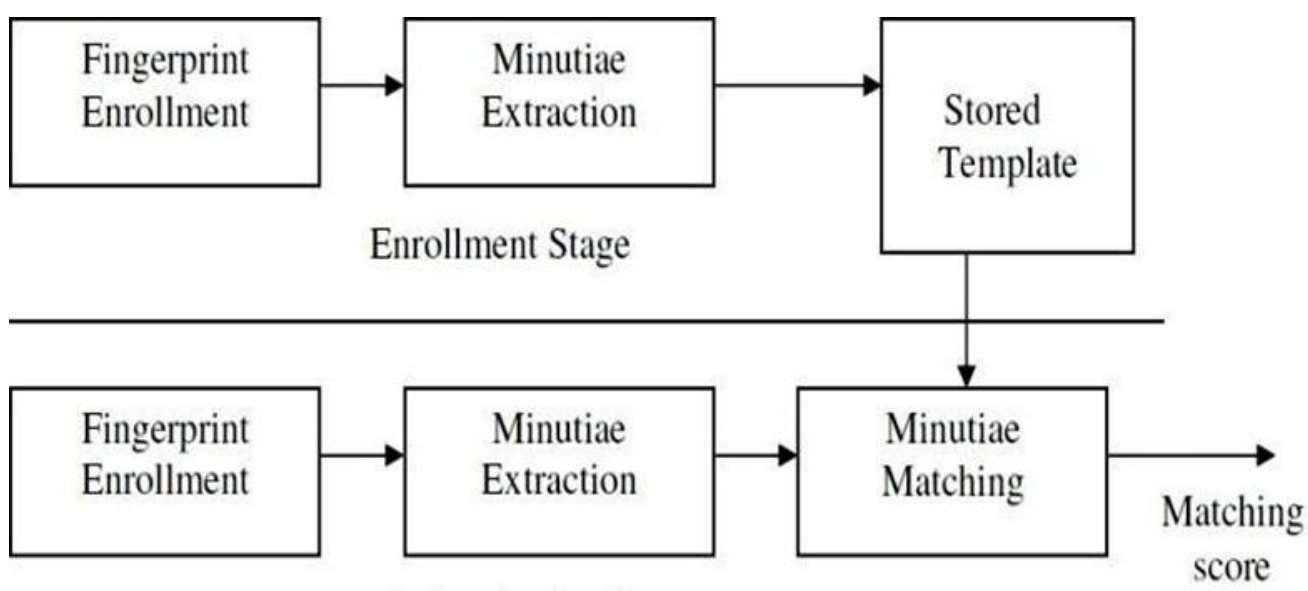

Authentication Stage 
Figure 2: Fingerprint authentication steps.

\section{Literature Survey}

The thought behind this is to catch the patterns in exactness and execution upgrade in multi biometric frameworks during this time of expanded biometric acknowledgment frameworks. Subsequent to surveying the techniques utilized by different analysts dependent on the format standards, the primary highlights of the chose strategies were resolved and afterward their benefits and deficiencies contributed as a feature of the audit. This investigation and correlation will toward the end be utilized to decide the holes in exactness and execution in the current multi biometric frameworks.

A. K. Jain [17] made us to comprehend that to beat a portion of the issues in unibiometric frameworks utilizing multi biometric frameworks, combination of data at various levels should be thought about, including sensor level, highlight extraction level, coordinating score level, and choice level. They additionally expressed that data combination at the component extraction level is far superior than the sensor level, coordinating score level and choice level, since it gives an invariant portrayal of the biometric design and the issue of clamor have been diminished.

K. Sasidhar [8] inspected the precision and execution of multimodal biometric validation frameworks utilizing the cutting edge Commercial Off-The-Shelf (COTS) items. Their point of utilizing a multi biometric framework was to diminish at least one of the issues experienced in unimodal biometric frameworks including (I) bogus acknowledge rate (FAR), (ii) bogus oddball rate (FRR), (iii) inability to enlist rate (FTE), and (iv) defenselessness to curios or emulates. They applied the basic whole combination rule for different standardization procedures in tending to the issue of unique classifier yield scores in various mix order frameworks.

Their trial results show that at a FAR of $0.1 \%$ the straightforward whole combination with min-max standardization has a veritable acknowledge rate (GAR) of $94.9 \%$, which is viewed as in a way that is better than that of face, $75.3 \%$ and unique mark, $83.0 \%$. It obviously shows that the utilization of straightforward whole combination and standardization strategies upgrades the exhibition essentially over the face or unique mark classifiers.

A. Ross and R. G. Jan [2] introduced an element level combination plan of both hand and face modalities to improve multimodal coordinating execution. This is because of the way that combination at the element level is a moderately understudied issue contrasted with different levels, including the coordinating score and the choice level which are widely contemplated. They examined combination at the element level, which was tried on three distinct situations: (I) combination of PCA and LDA coefficients of face (Figure 3), (ii) melding of LDA coefficients relating to the R, G, B channels of a face picture (Figure 4) and (iii) combination of face and hand modalities (Figure 5). The aftereffects of the test on the first situation show that the exhibition of the LDA-based matcher is a lot higher than that of PCA-based matcher. The aftereffect of the subsequent situation shows that the proposed conspire beats coordinating score level combination by a considerable edge, along these lines underscoring the meaning of the proposed strategies. In conclusion, in the third situation the presentation of the proposed combination plot was seen to be better than that of coordinating score level combination.

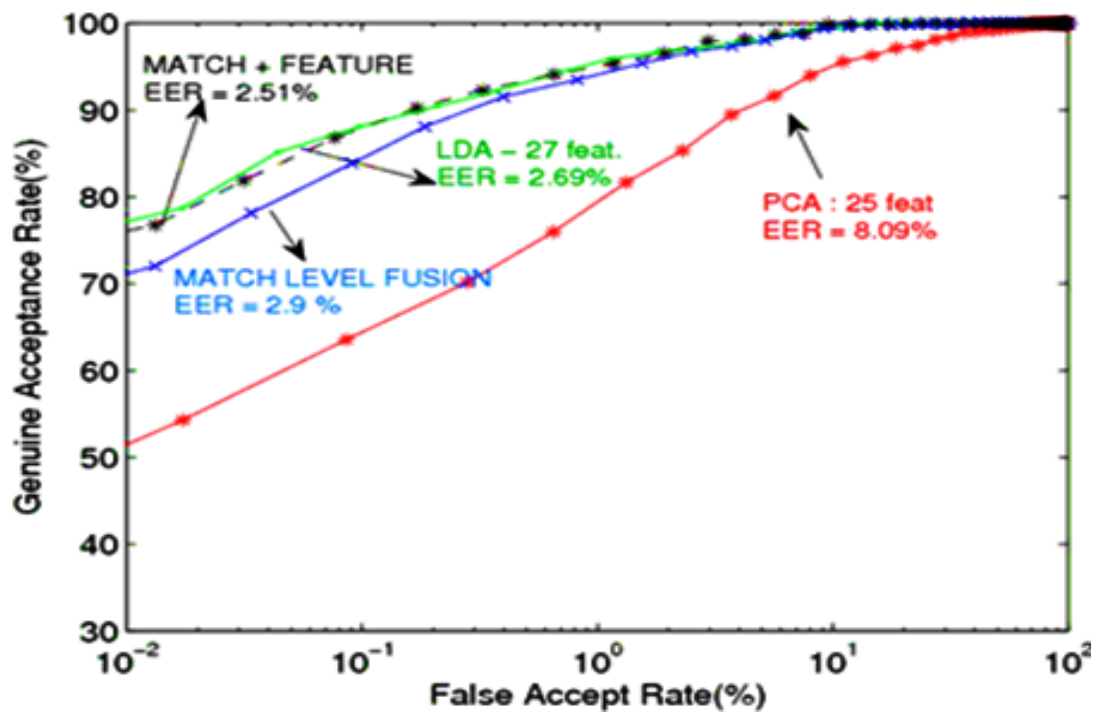

Figure 3: The consequences of combination when joining the PCA and LDA coefficients of dark scale face pictures 


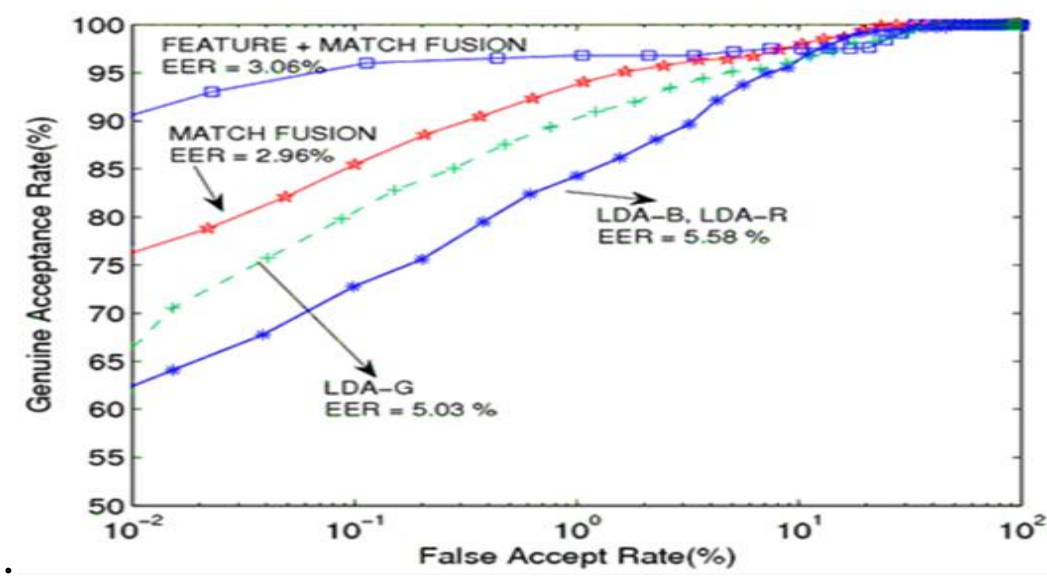

Figure 4: The aftereffects of combination when joining the LDA coefficients comparing to the R, G, Bchannels of a face picture.

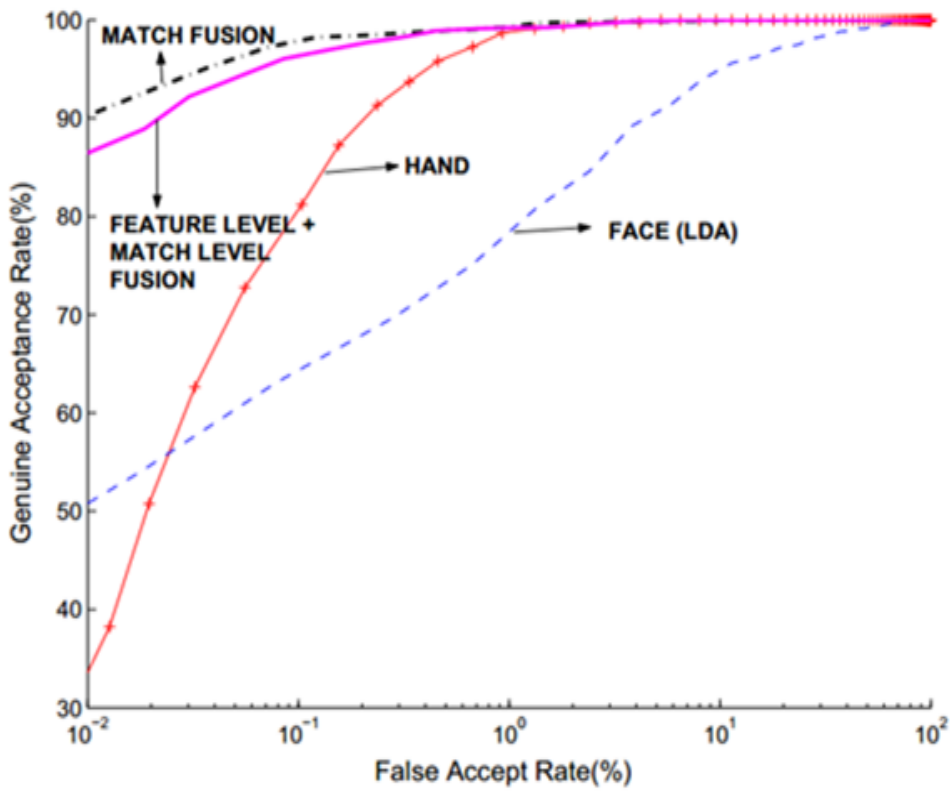

Figure 5: The results of fusion when combining the hand and face biometrics of a user.

N. Wang [9] proposed a novel multi biometrics combination strategy dependent on double iris, obvious and warm face modalities. The methodology highlights were melded at the component extraction level. At that point, the coordinating scores of iris and face modalities were combined utilizing three-sided standard. Their exploratory outcomes delineate that the multi biometrics combination accomplish higher distinguishing proof execution just as protection from satirizing assaults. At last, their proposed combination strategy beats the best in class technique.

U. Gawande [12] zeroed in on multimodal biometric framework utilizing the unique mark and iris modalities. Their proposed strategy extricated text based highlights of the two modalities utilizing the Haar wavelet-based method. At that point, the separated highlights were joined utilizing a novel element level combination calculation called Mahalanobis distance method. At long last, a Support Vector Machine (SVM) based learning calculation was utilized to prepare the framework. Their proposed technique demonstrated to have higher acknowledgment rate and more modest bogus dismissal pace of $7 \%$.

T. Ko [5] examined an outline on various potential techniques in multimodal biometric frameworks regarding their degrees of combination and the coordination methodologies that can be utilized to meld data and improve the general framework precision utilizing face, finger impression, and iris modalities. He spread the word about it that the main factors in deciding if a framework is all around planned and coordinated, are the framework's distinguishing proof precision and the need of staffing levels to appropriately work the framework. He further expressed that two normal systems typically utilized for intertwining face, finger impression, and iris classifiers are (I) to figure either an un-weighted or weighted measure of the planning distances and differentiation the distance with a breaking point, or (ii) to treat the organizing distance of various biometrics as a multi-dimensional 
component vector and use a classifier, for instance, the Fisher's separate examination or a neural association to describe the vector as being genuine or a trick.

R. Giot [11] introduced another multi biometrics approach utilizing combination capacities parameterized by different hereditary calculations utilizing a quick Error Equal Rate (EER) figuring technique to accelerate the wellness assessment. Their test results demonstrate that the produced capacities gives preferable outcomes over the whole, which is for the most part acknowledged as a decent combination work.

M Tiwari [18] Four classifiers are used in this examination, these are Bayesian association, multilayer perceptual neural association, $\mathrm{k}$ nearest neighbor and support vector machine. Besides, four intriguing preliminaries were used to test the classifier. These are cross-endorsed cases with $70 \%$ planning $30 \%$ testing, $60 \%$ getting ready $20 \%$ testing finally $60 \%$ planning $40 \%$ testing 10 covering cases. From the results we can surmise that all standard groupings of the classifiers complete a depiction speed of more than $90 \%$. Regardless, SVM is at this point the best recommended classifier for tally. Fingerprints are the most dependable confirmation are now true in court. As to inconceivable potential as a sensible approach for seeing fingers, this work attempts to investigate relationship between ladies. Appraisal and game-plan are the aftereffect of our figuring and a piece of the explanations behind seeing the right norms of sex.

A. J. Basha [10] clarified that the precision of biometric frameworks differs with the sort of modalities that are being utilized in it, although, a multimodal biometric framework was restricted to time imperatives because of its numerous handling stages. They presented a unique finger impression confirmation strategy intertwined with upgraded iris acknowledgment utilizing the versatile position level combination technique to improve the speed of verification with worthy exactness in the biometric framework. Their exploratory outcome shows that the framework improved in the False Acceptance Rate (FAR) and Equal Error Rate (EER) bends. At long last, the time spent for the preparation and confirmation stage was decreased by $10 \%$ contrasted and other framework.

In the audit talked about above, it was seen that diverse component extraction calculations were utilized for various modalities/qualities in the combination of two of more modalities. This adds to increment in season of execution because of their different handling stages and like insightful, limits the precision and execution of biometric acknowledgment frameworks.

\section{Proposed technique}

From the features in Table 1 we show the need of proposing another strategy for improving the precision and execution of multi biometric frameworks that will help connect the current holes. In this paper we propose a novel method of improving the exactness and execution in data combination of bimodal face and unique finger impression biometric framework utilizing Multilayer Perceptions (MLP) neural. Figure 4 outlines the engineering plan of the proposed technique. 


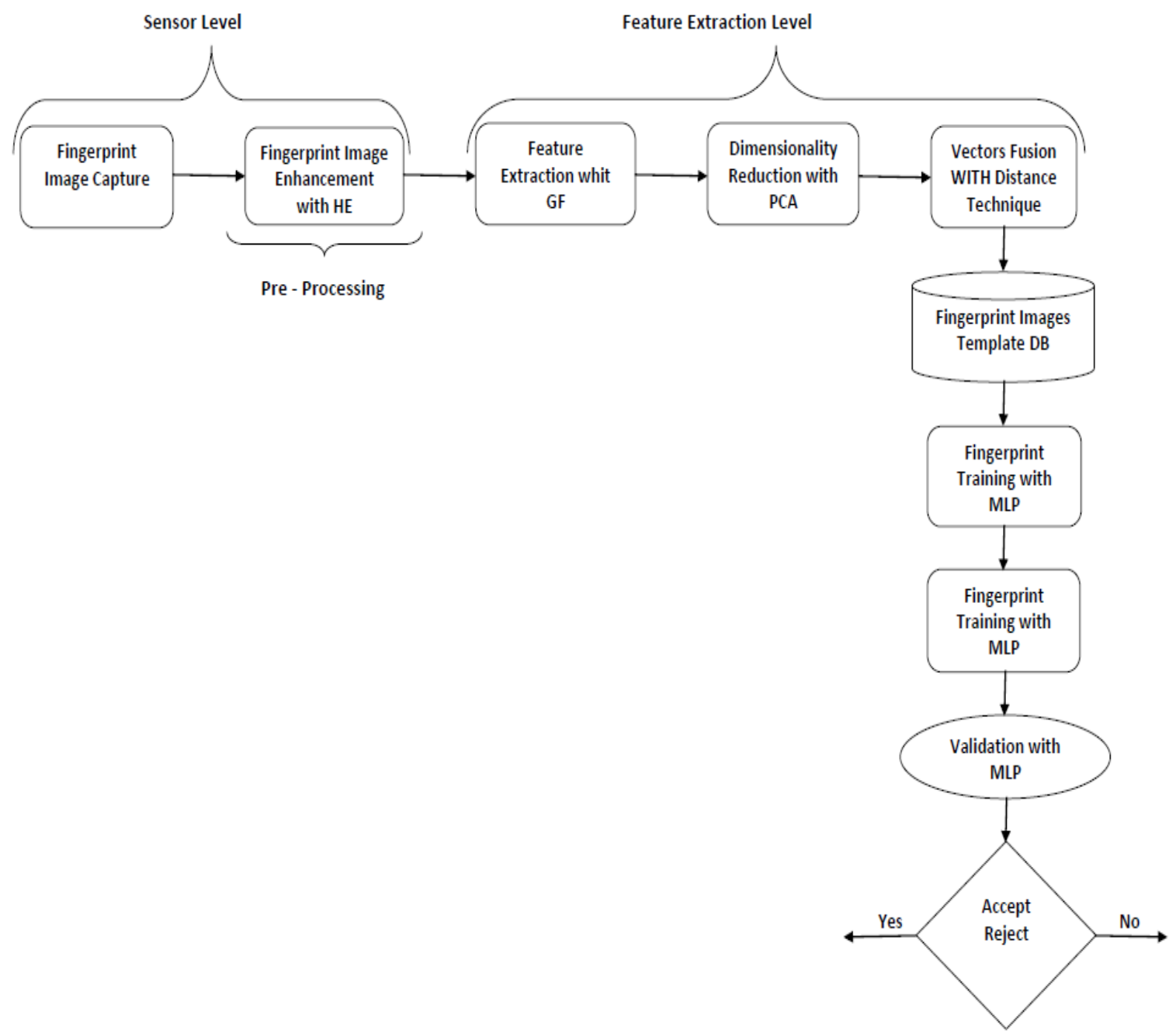

Figure 6: Architectural design of the proposed methodology

\section{Analysis and discussion}

The approaches introduced in there viewed papers zeroed in on tending to the precision and execution of multi biometric acknowledgment frameworks. Each work has contributed to some extent towards taking care of the current issues yet the principle challenge that is as yet experienced is the failure of the multi biometric frameworks to have an improved exactness and execution. Be that as it may, the greater part of these techniques are centered around various modalities. This outcomes into helpless combination of any consolidated modalities, because of the way that every methodology is actualized with an alternate calculation.

As talked about in the past areas, all strategies/methods have some novel and recognizable benefits towards addressing the difficulties looked in precision and execution of multi biometric frameworks. The benefits and faults of the strategies/procedures are featured in Table 1.

Table 1: Overview of Discussed Recognition Techniques in Multi biometric System

\begin{tabular}{|c|c|c|c|c|}
\hline Respect Techniques & Procedure & FAR & FRR & Benefits \\
\hline $\begin{array}{l}\text { PCA and LDA; Multi } \\
\text { algorithm ( A. Ross and } \\
\text { R. G. Jan, 2005) }\end{array}$ & $\begin{array}{l}\text { Face (feature } \\
\text { level) }\end{array}$ & 0.01 & 0.01 & $\begin{array}{l}\text { PCA outperforms LDA when the } \\
\text { training data set is small and less } \\
\text { sensitive to different training data } \\
\text { sets. LDA deals directly with } \\
\text { discrimination between classes. } \\
\text { Likewise, PCA tends to compute } \\
\text { vector that has the largest } \\
\text { variance associated with it. (M. } \\
\text { Aleix and A. C. Kak, 2001) }\end{array}$ \\
\hline Delaunay triangulation & Face & 8.07 & 2.95 & It lessens memory prerequisites \\
\hline
\end{tabular}




\begin{tabular}{|l|l|l|l|l|}
\hline A. Rattani et al., 2007) & $\begin{array}{l}\text { fingerprint } \\
\text { (matching } \\
\text { level and } \\
\text { Face and } \\
\text { fingerprint } \\
\text { (feature level) }\end{array}$ & 0.89 & 2.95 & $\begin{array}{l}\text { without forfeiting } \\
\text { acknowledgment precision, and } \\
\text { improves acknowledgment time. } \\
\text { It lessens memory prequisites } \\
\text { without forfeiting } \\
\text { acknowledgment precision, and } \\
\text { improves acknowledgment time. }\end{array}$ \\
\hline $\begin{array}{l}\text { LRVT ( N. Wang } \text { et al., } \\
\text { 2013) }\end{array}$ & $\begin{array}{l}\text { Dual, Iris, } \\
\text { visible and } \\
\text { thermal face } \\
\text { imagery }\end{array}$ & 0.47 & 0.39 & $\begin{array}{l}\text { It works well on small data set } \\
\text { with high level of accuracy. }\end{array}$ \\
\hline $\begin{array}{l}\text { SVM (V. Conti et al., } \\
\text { 2010) }\end{array}$ & $\begin{array}{l}\text { Iris and } \\
\text { fingerprint }\end{array}$ & 0 & 0.08 & $\begin{array}{l}\text { Extremely powerful non-linear } \\
\text { classifier }\end{array}$ \\
\hline $\begin{array}{l}\text { SVM ( U. Gawande et } \\
\text { al., 2013) }\end{array}$ & $\begin{array}{l}\text { Iris and } \\
\text { fingerprint }\end{array}$ & 0 & 0.06 & $\begin{array}{l}\text { It beats different strategies in } \\
\text { choosing the ideal hyper- } \\
\text { boundaries of a SVM when the } \\
\text { dimensionality of the information } \\
\text { is bigger than the quantity of tests } \\
\text { (D. Anguita et al., 2012) }\end{array}$ \\
\hline
\end{tabular}

\section{Conclusion and suggestion}

The appropriation of biometric acknowledgment frameworks will increment enormously soon because of the increment in the utilization of biometric frameworks (both unimodal and multimodal biometric frameworks). However, the issue of precision and execution is as yet a significant and intriguing viewpoint with regards to biometric acknowledgment frameworks to manage. In this audit, the strategies utilized by different analysts were talked about, the fundamental highlights of the chose techniques were resolved and afterward their qualities and inadequacies were called attention to. At that point, we proposed a novel methodology in combining both face and unique finger impression modalities utilizing a similar calculation (Gabor channel). At long last, a multilayer perception neural organization is utilized in the grouping measures, which by and large appears to be encouraging in expanding the degree of precision and execution in biometric acknowledgment frameworks whenever actualized..

\section{References}

L. Eugen and G. P. Petre, "Multimodal Biometric Systems Overview". ACTA TECHNICA NAPOCENSIS Electronics and Telecommunications. Volume 49, Number 3, 2008

A. A. Ross, and R. Govindarajan. "Feature Level Fusion Using Hand and Face Biometrics". In Defense and Security, pp. 196-204. International Society for Optics and Photonics, 2005.

A. Rattani, D. R. Kisku, M. Bicego and M. Tistarelli, "Feature Level Fusion of Face and Fingerprint Biometrics". Biometrics: Theory, Applications, and Systems, 2007. BTAS 2007. First IEEE International Conference on. IEEE, 2007.

A. K. Jain, K. Nandakumar, X. Lu, and U. Park "Integrating Faces, Fingerprints, and Soft Biometric Traits for User Recognition”. Biometric Authentication. Springer Berlin Heidelberg, 2004. 259-269.

T. Ko, "Multimodal Biometric Identification for Large User Population Using Fingerprint, Face and Iris Recognition". In Applied Imagery and Pattern Recognition Workshop, 2005. Proceedings. 34th, pp. 6-pp. IEEE, 2005.

Y. Elmir, O. Ghazaoui, and F. Boukenni, "Multimodal Biometric System's Resistance to noise: Fingerprint and Noise". Universite d'Adrar, Algerie, 2012

A. Jagadeesan and K. Duraiswamy, "Secured Cryptographic Key Generation from Multimodal Biometrics: Feature Level Fusion of Fingerprint and Iris" arXiv preprint arXiv:1003.1458 (2010).

K. Sasidhar, V. L. Kakulapati, K. Ramakrishna, and K. KailasaRao, "Multimodal Biometric Systems - Study to Improve Accuracy and Performance". arXiv preprint arXiv:1011.6220 (2010).

N. Wang, Q. Li, A. A. Abd El-Latif, J. Peng and X. Niu, "Multibiometrics Fusion for Identity Authentication: Dual Iris, Visible and Thermal Face Imagery". extraction 2 (2013): 2FPCA.

A. J. Basha, V. Palanisamy, and T. Purusothaman, "Efficient Multimodal Biometric Authentication Using Fast Fingerprint Verification and Enhanced Iris Features". Journal of Computer Science 7, no. 5 (2011): 698.

R. Giot, M. El-Abed, and C. Rosenberger, "Fast Learning For Multibiometrics Systems Using Genetic Algorithms" High Performance Computing and Simulation (HPCS), 2010 International Conference on. IEEE, 2010. 
U. Gawanda, M. Zaveri, and A. Kapur, "A Novel Algorithm for Feature Level Fusion Using SVM Classifier for Multibiometrics-Based Person Identification". Applied Computational Intelligence and Soft Computing, 2013, 9.

M. Aleix and A. C. Kak, "PCA versus LDA". Pattern Analysis and Machine Intelligence, IEEE Transactions on 23.2 (2001): 228-233.

U. M. Braga-Neto and E. R. Dougherty, "Is Cross-Validation Valid for Small Sample Micro Array Classification?”. Bioinformatics, vol. 20, pp. 374-380, 2004

A. Isaksson, M. Wallman, H. Goeransson and M.G. Gustafsson, "Cross-Validation and Bootstrapping are Unreliable in Small Sample Classification”. Pattern Recognition Letters. vol. 29, pp. 1960-1965, 2008

R. Collobert and S. Bengio, "Links between Perceptrons, MLPs and SVMs". Proceedings of the twenty-first international conference on Machine learning. ACM, 2004.

A. K. Jain, A. A. Ross, and K. Nandakumar, "Introduction to Biometrics". Springer, 2011.

Meena Tiwari, V. Shanthi, and Ashish Mishra, "Development of Association Rule Mining Model for Gender Classification”2021 IOP Conf. Ser.Mater. Sci. Eng.1022 01206 